Pacific Journal of Mathematics

CLOSED VECTOR FIELDS

September 1965 


\title{
CLOSED VECTOR FIELDS
}

\author{
N. HICKS
}

We study closed vector fields on a semi-Riemannian manifold. In particular, we study the differential geometry of the submanifolds determined by a nonvanishing closed field. Expressions are computed for the Weingarten map, the mean curvature, the Riemannian curvature, and the Laplacian of the square of the length of the field. Thus we obtain a necessary and sufficient condition that the constant hypersurface of a nontrivial harmonic function be a minimal surface. We obtain conditions that imply the classical Codazzi-Mainardi equations hold. We obtain conditions that imply the existence of a representation of the manifold as a cross product in which one factor is a real line. Finally, various special cases are examined.

1. Notation. Let $M$ be a connected $C^{\infty}$ semi-Riemannian manifold with metric tensor $\langle$,$\rangle and Riemannian connexion D$ [see Helgason 4 or Hicks 7 for definitions]. We summarize the properties of $D$ and some associated concepts we shall use. The operator $D$ assigns to each pair of $C^{\infty}$ vector fields $X$ and $Y$ on an open set $U$ of $M$, a $C^{\infty}$ vector field $D_{X} Y$ called the covariant derivative of $Y$ in the direction $X$. If $X, Y$, and $Z$ are $C^{\infty}$ fields on $U$ and $f$ a $C^{\infty}$ function (real valued) on $U$ then we have the following relations between vector fields on $U$ :

$$
\begin{aligned}
D_{X}(Y+Z) & =D_{X} Y+D_{X} Z \\
D_{(X+Y)} Z & =D_{X} Z+D_{Y} Z \\
D_{f X} Y & =f D_{X} Y \\
D_{X}(f Y) & =(X f) Y+f D_{X} Y \\
\operatorname{Tor}(X, Y) & =D_{X} Y-D_{Y} X-[X, Y] \\
R(X, Y) Z & =D_{X} D_{Y} Z-D_{Y} D_{X} Z-D_{[X, Y]} Z .
\end{aligned}
$$

We call Tor the torsion on $D$ and $R$ the curvature of $D$. Since $D$ is Riemannian, Tor $=0$, and $D$ is compatible with the metric tensor, thus

$$
\begin{aligned}
D_{X} Y-D_{Y} X & =[X, Y] \\
X\langle Y, Z\rangle & =\left\langle D_{X} Y, Z\right\rangle+\left\langle Y, D_{X} Z\right\rangle .
\end{aligned}
$$

We extend the operator $D_{x}$, as usual, to be a complete derivation on the tensor algebra over $M$. If $T^{r, s}$ denotes the set of $r$-contravariant and $s$-covariant tensors on $M$, then $D_{X}: T^{r, s} \rightarrow T^{r, s}$. If $f \in T^{0,0}$, then $D_{X} f=X f$. If $Y \in T^{1,0}$, then $D_{X} Y$ is given by the connexion. If

Received January 16, 1964. This work was supported in part by NSF Grants G 23842 and GP 88. 
$w \in T^{0,1}$, then $\left(D_{X} w\right)(Y)=X(w(Y))-w\left(D_{X} Y\right)$. The last equality contains the seeds of what is meant by a complete derivation which we explain. Having defined $D_{x}$ on functions, fields, and 1-forms if $\phi \in T^{r, s}, w_{i} \in T^{0,1}$ for $i=1, \cdots, r$, and $Y_{j} \in T^{1,0}$ for $j=1, \cdots, s$, then

$$
\begin{aligned}
& X \phi\left(w_{1}, \cdots, w_{r}, Y_{1}, \cdots, Y_{s}\right)=\left(D_{X} \phi\right)\left(w_{1}, \cdots, w_{r}, Y_{1}, \cdots, Y_{s}\right) \\
& \quad+\sum_{i} \phi\left(w_{1}, \cdots, w_{i-1}, D_{X} w_{i}, w_{i+1}, \cdots, w_{r}, Y_{1}, \cdots, Y_{s}\right) \\
& \quad+\sum_{j} \phi\left(w_{1}, \cdots, w_{r}, Y_{1}, \cdots, Y_{j-1}, D_{X} Y_{j}, Y_{j+1}, \cdots, Y_{s}\right)
\end{aligned}
$$

where all terms are well-defined except the first term on the right side of the equation.

The symbol $\Delta$ will denote the general covariant differentiation operator $4: T^{r, s} \rightarrow T^{r, s+1}$ which is induced by $D$. Using the above notation, $(\Delta \phi)\left(w_{1}, \cdots, w_{r}, Y_{1}, \cdots, Y_{r}, X\right)=\left(D_{X} \phi\right)\left(w_{1}, \cdots, w_{r}, Y_{1}, \cdots, Y_{s}\right)$.

Our study will concern linear transformation valued tensors on $M$ (tensor fields of type 1,1). For completeness, we define a linear transformation valued tensor $A$ on an open set $U$ of $M$ to be a mapping that assigns to each point $m$ in $U$, a linear transformation $A_{m}: M_{m} \rightarrow$ $M_{m}$, where $M_{m}$ is the tangent space at $m$. We say $A$ is $C^{\infty}$ if it maps $C^{\infty}$ fields on $U$ into $C^{\infty}$ fields; then if $X$ is a $C^{\infty}$ field on $U$ then the field $(A(X))_{m}=A_{m}\left(X_{m}\right)$ is $C^{\infty}$ on $U$. We define the vector valued 2-form $\mathrm{Tor}_{A}$ by

$$
\operatorname{Tor}_{A}(X, Y)=D_{X} A(Y)-D_{Y} A(X)-A[X, Y]
$$

and let $\operatorname{tr} A$ and $\operatorname{det} A$ denote the trace and determinant functions on $A$, respectively.

We will use $G$ to denote the nonsingular linear transformation induced by the metric tensor that maps $M_{m}$ onto $M_{m}^{*}$ for each $m$. Thus if $X$ is in $M_{m}$ then $G(X)(Y)=\langle X, Y\rangle$ for $Y$ in $M_{m}$; or $G(X)=$ $C_{X}\langle\rangle=,\langle X$,$\rangle where C_{X}$ is contraction by $X$ in the first covariant slot. We also use the symbol $G$ for the inverse of $G$. Thus we think of $G$ as a "switch map" and let the argument it is applied to tell us which map is being used. A vecter field $X$ will be called closed (or exact) if $G(X)$ is closed (or exact), and $X$ is geodesic if $D_{X} X=0$. A vector $X$ is nonsingular (not light-like) if $\langle X, X\rangle \neq 0$. If $\theta \in T^{r, s}$ with $r>0$, then the divergence of $\theta$ is the tensor $\operatorname{div} \theta \in T^{r-1, s}$ defined by $\operatorname{div} \theta=\operatorname{tr} \Delta \theta$, where the trace is taken on the last covariant slot and last contravariant slot. If $Z_{1}, \cdots, Z_{n}$ is a base field of independent $C^{\infty}$ vector fields on an open set $U$ in $M$ and $z_{1}, \cdots, z_{n}$ is the dual base of 1 -forms, then

$$
\begin{aligned}
& (\operatorname{div} \theta)\left(w_{1}, \cdots, w_{r-1}, Y_{1}, \cdots, Y_{s}\right) \\
& \quad=\sum_{j=1}^{n}(\Delta \theta)\left(w_{1}, \cdots, w_{r-1}, z_{j}, Y_{1}, \cdots, Y_{s}, Z_{j}\right) .
\end{aligned}
$$


If $f \in T^{0,0}$, then the gradient of $f$, grad $f$, is the vector field $G(d f)$, so 〈grad $f, X\rangle=X f$, and the Laplacian of $f, A_{2} f$, is the function $\operatorname{div}(\operatorname{grad} f)$. A function $f$ is harmonic if $\Delta_{2} f=0$, and a field $T$ is conservative if $\operatorname{div} T=0$.

2. Operators associated with a vector field. Let $T$ be a $C^{\infty}$ vector field on $M$. On each tangent space $M_{m}$, we define linear maps $A_{T}, B_{T}$, and $C_{T}$ by

$$
A_{T}(X)=D_{X} T, B_{T}(X)=D_{X}\left(D_{T} T\right), \quad \text { and } \quad C_{T}(X)=R(X, T) T .
$$

These maps are $C^{\infty}$ since $D$ and $T$ are $C^{\infty}$. Let $U$ be the open set of points in $M$ where $\langle T, T\rangle$ does not vanish. On $U$, we define the $C^{\infty}(n-1)$ dimensional distribution $T^{\perp}$ by

$$
\left(T^{\perp}\right)_{p}=\left[X \in M_{p}:\langle X, T\rangle=0\right] .
$$

From the definition of the curvature $R$ we have

$$
C_{T}=B_{T}-A_{T}^{2}+\left[A_{T}, D_{T}\right]
$$

where

$$
\left[A_{T}, D_{T}\right](X)=A_{T}\left(D_{T} X\right)-D_{T}\left(A_{T} X\right)
$$

and thus $\left[A_{T}, D_{T}\right]$ is a linear transformation valued tensor. By the standard symmetry properties of the four covariant Riemann Christoffel tensor, the map $C_{T}$ is symmetric (self-adjoint), and we call it the Ricci map associated with $T$. The trace of $C_{T}$ is the Ricci curvature of $T$, which we denote by $\operatorname{Ric}(T, T)$.

Following Bochner [1], we say a field $T$ is restrained if $\Delta_{2}\langle T, T\rangle<0$ at some point or $T$ has constant length. Bochner has shown that every field on a compact manifold is restrained, and in the noncompact case, a field is restrained if its length attains a relative maximum at some point.

Our main interests in this study are the cases when $A_{T}$ is symmetric, or equivalently, $T$ is closed. Since the gradient of any $C^{\infty}$ function is a closed field, many closed fields exist.

Proposition 1. For any field $T, \operatorname{tr} A_{T}=\operatorname{div} T$ and $\operatorname{tr}\left[A_{T}, D_{T}\right]=$ $-T(\operatorname{div} T)$. If $T=\operatorname{grad} f$, then the Laplacian of $f$ is the trace of $A_{T}$.

Proof. Let $Z_{1}, \cdots, Z_{n}$ be a set of nonsingular orthonormal vector fields belonging to a Riemannian normal coordinate system at a point $m$ in $M$ and let $w_{1}, \cdots, w_{n}$ be the dual 1 -forms of this base. Thus if $e_{i}=\left\langle Z_{i}, Z_{i}\right\rangle$, then

$$
\operatorname{tr} A_{T}=\Sigma e_{i}\left\langle D_{z_{i}} T, Z_{i}\right\rangle=\Sigma w_{i}\left(D_{Z_{i}} T\right)=\operatorname{tr} \Delta(T),
$$


and using the fact that $D_{T} Z_{i}=0$ at $m$ for any $T$,

$$
\begin{aligned}
\operatorname{tr}\left[A_{T}, D_{T}\right] & =\Sigma\left\langle A_{T} D_{T} Z_{i}-D_{T} A_{T} Z_{i}, Z_{i}\right\rangle e_{i} \\
& =\Sigma\left\langle D_{D_{T} Z_{i}} T-D_{T} D_{Z_{i}} T, Z_{i}\right\rangle e_{i} \\
& =-\Sigma T\left\langle D_{Z_{i}} T, Z_{i}\right\rangle e_{i}+\Sigma\left\langle D_{Z_{i}} T, D_{T} Z_{i}\right\rangle e_{i} \\
& =-T\left(\operatorname{tr} A_{T}\right) .
\end{aligned}
$$

Proposition 2. For any field $T$,

$$
\operatorname{Ric}(T, T)=\operatorname{tr} C_{T}=\operatorname{tr} B_{T}-\operatorname{tr} A_{T}^{2}-T(\operatorname{div} T) .
$$

Proof. Using the fields $Z_{i}$ in the above proof,

$$
\operatorname{tr} C_{T}=\Sigma\left\langle R\left(Z_{i}, T\right) T, Z_{i}\right\rangle e_{i}=\operatorname{Ric}(T, T),
$$

and the rest of the proposition follows from the linearity of the trace.

Proposition 3. For any field $T, T$ has constant length if and only if (Image $\left.A_{T}\right) \subset T^{\perp}$.

Proof. For any vector $X$,

$$
X\langle T, T\rangle=2\left\langle D_{X} T, T\right\rangle=2\left\langle A_{T}(X), T\right\rangle .
$$

3. The symmetric case. Throughout this section we assume $T$ is a closed field, or equivalently, $A_{T}$ is symmetric (by the following proposition).

Theorem 1. A field $T$ is closed if and only if $A_{T}$ is symmetric. If $T$ is closed, then $T^{\perp}$ is integrable on $U$.

Proof. If $X$ and $Y$ are fields, then

$$
\begin{aligned}
(d G(T))(X, Y) & =X\langle T, Y\rangle-Y\langle T, X\rangle-\langle T,[X, Y]\rangle \\
& =\left\langle D_{X} T, Y\right\rangle-\left\langle D_{Y} T, X\right\rangle+\left\langle T, D_{X} Y-D_{Y} X-[X, Y]\right\rangle \\
& =\left\langle A_{T} X, Y\right\rangle-\left\langle A_{T} Y, X\right\rangle,
\end{aligned}
$$

since the torsion of $D$ is zero.

If $X$ and $Y$ belong to $T^{\perp}$, then

$$
\begin{aligned}
\langle[X, Y], T\rangle & =\left\langle D_{X} Y-D_{Y} X, T\right\rangle \\
& =X\langle Y, T\rangle-\left\langle Y, D_{X} T\right\rangle-Y\langle X, T\rangle+\left\langle X, D_{Y} T\right\rangle \\
& =\left\langle X, A_{T} Y\right\rangle-\left\langle Y, A_{T} X\right\rangle=0
\end{aligned}
$$

since $\langle Y, T\rangle \equiv\langle X, T\rangle \equiv 0$. Thus $T^{\perp}$ is involutive or integrable (see Chevalley [2]). 
In the special case $T=\operatorname{grad} f$, then the integral manifolds of $T^{\perp}$ on $U$ are precisely the hypersurfaces on which $f$ is constant. We next investigate the geometry of an integral manifold $M^{\prime}$ of $T^{\perp}$ through a point $m$ in $U$. Since $T$ is normal to $M^{\prime}$, we use $T$ to frame $M^{\prime}$ locally (see Hicks [6]). Let $e$ be the function on $U$ which is plus or minus one according as $\langle T, T\rangle$ is positive or negative, respectively.

THeOREM 2. Let $L$ be the Weingarten map on $M^{\prime}$ and take $X$ in $\left(M^{\prime}\right)_{m}$.

$$
L(X)=[e\langle T, T\rangle]^{-3 / 2}\left[e\langle T, T\rangle A_{T}(X)-e\left\langle T, A_{T}(X)\right\rangle T\right]
$$

and the mean curvature $H$ of $M^{\prime}$ is given by

$$
H=\operatorname{tr} L=|T|^{-1}[\operatorname{div} T-T \log |T|]
$$

where $|T|=[e\langle T, T\rangle]^{1 / 2}$ is the length of $T$. Thus $M^{\prime}$ is minimal if and only if $\operatorname{div} T=T \log |T|$.

Proof. Let $N=[e\langle T, T\rangle]^{-1 / 2} T$ be the unit normal so

$$
L(X)=D_{X} N=-[e\langle T, T\rangle]^{-3 / 2} e\left\langle A_{T} X, T\right\rangle T+[e\langle T, T\rangle]^{-1 / 2} A_{T} X .
$$

To compute $\operatorname{tr} L$, let $Z_{1}, \cdots, Z_{n-1}$ be a nonsingular orthonormal base of $\left(M^{\prime}\right)_{m}$ and let $Z_{n}=N$. Letting $e_{i}=\left\langle Z_{i}, Z_{i}\right\rangle$, then

$$
\begin{aligned}
H=\operatorname{tr} L & =\sum_{j=1}^{n-1}\left\langle L Z_{j}, Z_{j}\right\rangle e_{j} \\
& =[e\langle T, T\rangle]^{-1 / 2} \sum_{1}^{n-1}\left\langle A_{T} Z_{j}, Z_{j}\right\rangle e_{j} .
\end{aligned}
$$

But

$$
\begin{aligned}
\left\langle A_{T} Z_{n}, Z_{n}\right\rangle e_{n} & =\left\langle D_{N} T, N\right\rangle e=\left\langle D_{T} T, T\right\rangle\langle T, T\rangle \\
& =(1 / 2)(T\langle T, T\rangle)\langle T, T\rangle=(1 / 2) T \log e\langle T, T\rangle .
\end{aligned}
$$

Hence, $H=(e\langle T, T\rangle)^{-1 / 2}\left[\operatorname{tr} A_{T}-T \log |T|\right]$.

COROLLARY 1. The constant hypersurfaces of a nonconstant harmonic function are minimal surfaces if and only if the gradient of the function has constant length along its integral curves.

Proof. Let $f$ be harmonic and $T=\operatorname{grad} f$. Then $T$ is closed and $\operatorname{tr} A_{T}=\operatorname{div} T=0$. Hence $H=0$ if and only if $\left\langle D_{T} T, T\right\rangle=0$ or $T\langle T, T\rangle=0$.

CoRollary 2. Let $T$ be a unit field on $M$ which is closed. Then 
the total curvature and mean curvature of the integral manifolds of $T^{\perp}$ are given by $K=\operatorname{det} A_{T}$ and $H=\operatorname{div} T . \quad$ Indeed, $S=A_{T}$ if and only if $T$ is a unit field.

The first corollary above suggests the definition of a minimal harmonic function as a harmonic function whose constant hypersurfaces are minimal surfaces. This class of harmonic functions has not been examined as yet, as far as we know, nor has the above result (Corollary 1) been proven before.

Proposition 4. Let $\phi=\langle T, T\rangle$. Then $\operatorname{grad} \phi=2 D_{T} T$, which implies $B_{T}$ is symmetric, and

$$
\Delta_{2} \phi=2 \operatorname{tr} B_{T}=2\left[\operatorname{Ric}(T, T)+\operatorname{tr} A_{T}^{2}+T(\operatorname{div} T)\right]
$$

while

$$
\left(\Delta^{2} \phi\right)(Z, Y)=2\left\langle B_{T} Z, Y\right\rangle
$$

Proof. Consider

$$
(\Delta \phi) X=X\langle T, T\rangle=2\left\langle D_{X} T, T\right\rangle=2\left\langle X, D_{T} T\right\rangle .
$$

Hence $\operatorname{grad} \phi=2 D_{T} T$, and $A_{2} \phi=\operatorname{div} \operatorname{grad} \phi=2 \operatorname{tr} B_{T}$. The last expression for the Laplacian of $\phi$ follows from Proposition 2.

Finally,

$$
\left(\Delta^{2} \phi\right)(Z, Y)=\left[D_{Y}(\Delta \phi)\right] Z=2 Y\left\langle Z, D_{T} T\right\rangle-2\left\langle D_{Y} Z, D_{T} T\right\rangle=2\left\langle Z, B_{T} Y\right\rangle .
$$

We have immediately a slight generalization of a result of Bochner [1].

COROLlary 1. Let $T$ be a closed field such that $\operatorname{div} T$ is constant along the integral curves of $T$. If $T$ is restrained, then $\operatorname{Ric}(T, T)<0$ at some point of $M$ or $\operatorname{Ric}(T, T) \leqq 0$ on all of $M$. On a compact manifold whose Ricci curvature is always positive there can be no nontrivial closed field $T$ with $T(\operatorname{div} T)=0$. On a compact manifold whose Ricci curvature is nonnegative any nontrivial closed field $T$ with $T(\operatorname{div} T)=0$ must be a global parallel field with constant length, zero Ricci curvature, and $A_{T}=0$ (see Proposition 6).

Proof. In these cases,

$$
\operatorname{Ric}(T, T)=(1 / 2) \Delta_{2} \phi-\operatorname{tr} A_{T}^{2}
$$

which proves the first two statements immediately. If $T$ is restrained, as in the last statement, then we force $\operatorname{Ric}(T, T) \equiv 0$ and $T$ to have constant length since $R(T, T)<0$ at any point is impossible. Thus $\phi$ 
is constant, $\Delta_{2} \phi=0$, and $\operatorname{tr} A_{T}^{2}=0$ which implies all the eigenvalues of $A_{T}$ are zero, so $A_{T}=0$.

Corollary 2. A nontrivial closed field has constant length on a semi-Riemannian manifold if and only if its integral curves are geodesics.

Proof. This is trivial since $\operatorname{grad} \phi=2 D_{T} T$.

The following result applies to any vector field.

Proposition 5. The integral curves of a field $T$ are reparameterizations of geodesics if and only if $D_{T} T=g T$ for some real valued $C^{\infty}$ function $g$.

Proof. If the field $f T$ is geodesic ( $f$ never vanishes), $0=D_{f T} f T=$ $f\left[(T f) T+f D_{T} T\right]$ and $g=-T(\log f)$. Conversely, if $D_{T} T=g T$ then along each integral curve of $T$ we need only solve the linear equation $(T f)+f g=0$ to obtain $f$ for which $f T$ is geodesic.

Corollary. If $T$ is closed, nonvanishing, and $D_{T} T=g T$ then $\operatorname{Ric}(T)=g \operatorname{div} T-\operatorname{tr} A_{T}^{2}+T(g-\operatorname{div} T)$.

We now study the case when $T$ has constant length on the hypersurfaces $M^{\prime}$.

THEOREM 3. The following four statements are equivalent on the set $U$ :

(a) $A_{T}$ is invariant on $T^{\perp}$.

(b) $T$ has constant length on any $M^{\prime}$.

(c) $D_{T} T$ is orthogonal to $T^{\perp}$.

(d) $[T, X]$ is in $T^{\perp}$ if $X$ in $T^{\perp}$.

Proof. If $X$ is in $T^{\perp}$ then $X\langle T, T\rangle=2\left\langle A_{T} X, T\right\rangle=2\left\langle X, D_{T} T\right\rangle$ which shows (a), (b), and (c) are equivalent. Also

$$
\begin{aligned}
\left\langle A_{T} X, T\right\rangle & =\left\langle X, A_{T} T\right\rangle=T\langle X, T\rangle-\left\langle D_{T} X, T\right\rangle \\
& =-\left\langle D_{X} T+[T, X], T\right\rangle,
\end{aligned}
$$

where we extend $X$ to be a $C^{\infty}$ field in $T^{\perp}$. Hence $2\left\langle A_{T} X, T\right\rangle=$ $\langle[X, T], T\rangle$ which shows (a) is equivalent to (d).

THEOREM 4. If one of the statements in Theorem 3 holds and T does not vanish, then the integral curves of $T$ are reparameterizations of geodesics, $\operatorname{grad} \phi=2 D_{T} T=(T \log e \phi) T$, and the vector $\operatorname{grad} \phi$ has 
constant length on $M^{\prime}$, i.e. $T \log \phi$ is constant on $M^{\prime}$. Moreover, the mean curvature of $M^{\prime}$ is constant if and only if $\operatorname{div} T$ is constant on $M^{\prime}$.

Proof. Letting $\operatorname{grad} \phi=f T$ then $T \phi=2\left\langle D_{r} T, T\right\rangle=\langle f T, T\rangle=$ $f \phi$. If $T \neq 0$, then $\phi \neq 0$, so $f=(T \phi) / \phi=T \log e \phi$. The integral curves. of $T$ are reparameterizations of geodesic by Proposition 5 .

Letting $X$ be a $C^{\infty}$ field in $T^{\perp}$, then

$$
X f=X T(\log \phi)=[X, T] \log \phi+T(X \log \phi)=0
$$

since $[X, T]$ is in $T^{\perp}$ and $\phi$ is constant on $M^{\prime}$.

The last statement of the conclusion follows from Theorem 2.

COROLLARY. If grad $\phi$ does not vanish on $M$, then the hypersurfaces $M^{\prime}$ are precisely the constant hypersurfaces of $\phi$ if and only if one of the statements in Theorem 3 is true.

We return to the study of the geometry of the hypersurface $M^{\prime}$. Recall the fact that if $L$ is the Weingarten map of an oriented nonsingular hypersurface in a semi-Riemannian manifold, then the Codazzi-Mainardi equations hold on the hypersurface if and only if Tor $_{L}=0$. In the following theorem, we write $A_{T}=\Delta T$ which is. admissable by the identification of linear transformations with tensors. of type 1,1 .

THEOREM 5. On the set $U$, the following three statements are equivalent:

(a) The Codazzi-Mainardi equations hold on $M^{\prime}$.

(b) Tor $_{\Delta T}=0$ on vectors in $T^{\perp}$.

(c) $R(X, Y) T=0$ for all $X, Y$ in $T^{\perp}$.

Proof. Let $D^{\prime}$ be the induced Riemannian covariant differentiation on $M^{\prime}$, thus for fields $X$ and $Y$ in $T^{\perp}$,

$$
D_{X} Y=D_{X}^{\prime} Y-\langle L X, Y\rangle r N
$$

by the Gauss equation (see Hicks [7]), where $r=\langle N, N\rangle=e$.

Using the Gauss equation and Theorem 2, a straightforward computation yields,

$$
\begin{aligned}
\operatorname{Tor}_{L}(X, Y)= & D_{X}^{\prime}(L Y)-D_{Y}^{\prime}(L X)-L([X, Y]) \\
= & {[e\langle T, T\rangle]^{-1 / 2} \operatorname{Tor}_{\Delta T}(X, Y) } \\
& -[e\langle T, T\rangle]^{-3 / 2} e\left\langle\operatorname{Tor}_{\Delta T}(X, Y), T\right\rangle T \\
= & |T|^{-1} \operatorname{Tor}_{\Delta T}(X, Y),
\end{aligned}
$$


since $\operatorname{Tor}_{\Delta T}(X, Y)=D_{X} A_{T} Y-D_{Y} A_{T} X-A_{T}[X, Y]=R(X, Y) T$ and $\langle R(X, Y) T, T\rangle=0$ by the skew-symmetry of the covariant RiemannChristoffel curvature tensor. Thus $\operatorname{Tor}_{\Delta T}(X, Y)$ has no component orthogonal to $M^{\prime}$ and the conclusion now follows.

THEOREM 6. On the set $U$, let $P$ be a two dimensional subspace of $M^{\prime}$ with nonsingular orthonormal base $X, Y$ Then

$$
\begin{aligned}
K(P)= & K^{\prime}(P) \\
& -[e\langle T, T \times X, X \backslash Y Y, Y\rangle]^{-1}\left[\left\langle A_{T} X, X \backslash A_{T} Y, Y\right\rangle-\left\langle A_{T} X, Y\right\rangle^{2}\right]
\end{aligned}
$$

relates the Riemannian curvature of $P$ with respect to $M$ and $M^{\prime}$.

Proof. The general Gauss curvature equation (see Hicks [6]) states that

$$
\tan R(X, Y) Z=R^{\prime}(X, Y) Z-r(\langle L Y, Z\rangle L X-\langle L X, Z\rangle L Y) .
$$

Using Theorem 2, a straightforward computation yields the result.

Corollary. If $M$ is Riemannian and $T=\operatorname{grad} f, m$ in $U$, and $x, y, \cdots$ are a set of Riemann normal coordinates at $m$ such that $\partial / \partial x$ and $\partial / \partial y$ span the subspace $P$ in $M_{m}^{\prime}$, then

$$
K(P)=K^{\prime}(P)-\left[\frac{\partial^{2} f}{\partial x^{2}} \frac{\partial^{2} f}{\partial y^{2}}-\left(\frac{\partial^{2} f}{\partial x \partial y}\right)^{2}\right] /|\operatorname{grad} f|^{2}
$$

at $m$.

Proof. Let $X=\partial / \partial x$ and $Y=\partial / \partial y$. Then $\left\langle A_{T} X, Y\right\rangle_{m}=\left\langle D_{X} T, Y\right\rangle=$ $X\langle T, Y\rangle=X_{m}(Y f)$ since $\left(D_{X} Y\right)_{m}=0$.

We now show the tensor Tor $_{\Delta T}$ represents a condition on the holonomy of the distribution $T^{\perp}$.

THEOREM 7. Let $M$ be Riemannian, complete, connected, and simply connected. Let $T$ be a nonvanishing closed field such that $\Delta T$ has no torsion. Then $M$ is diffeomorphic to a product $M^{\prime} \times R$, where $M^{\prime}$ is the $(n-1)$ dimensional integral submanifold of $T^{\perp}$ through a point $m$ in $M$ and $R$ is the real line. Hence the orbit space $M / T$ is diffeomorphic to $M^{\prime}$.

Proof. Since $M$ is simply connected its restricted homogeneous holonomy group is equal to its homogeneous holonomy group $H$. The Lie algebra of $H$ is generated by the linear transformations $R(X, Y)$ on $M_{m}$ for all vectors $X$ and $Y$ in $M_{m}$ (see Nomizu [8]). Since Tor $_{\Delta T}=$ $0, R(X, Y) T=0$ for all $X$ and $Y$ hence $R(X, Y)$ is invariant on $T^{\perp}$. 
Since $H$ is contained in the special orthogonal group $\mathrm{SO}(n, R)$, which is compact, the exponential map is onto. If $h$ is in $H$, then $h=$ $\exp R(X, Y)$ for some $X$ and $Y$ in $M_{m}$, and thus $h\left(T^{\perp}\right)$ is contained in $T^{\perp}$. We now apply the result of DeRham [3] to get $M=M^{\prime} \times N$. Since $M$ is Riemannian and complete, $N$ is diffeomorphic to the real line or the one dimensional torus. Since $M$ is simply connected, $N$ is diffeomorphic to $R$.

4. Special cases. We conclude with some special cases that follow from the above results. We will always assume the field $T$ is nontrivial, nonsingular, and closed.

Proposition 6. If $A_{T} \equiv 0$, then $T$ is a geodesic field with constant length, zero divergence, and zero Ricci curvature. If $T$ lies in the plane section $P$ then $K(P)=0$. Thus there is no pair of conjugate points along the geodesics determined by $T$. The distribution $T^{\perp}$ is integrable and its integral manifolds $M^{\prime}$ are flatly imbedded in $M$ (i.e. $L \equiv 0$ on $M^{\prime}$ ). Hence $M^{\prime}$ is a geodesic submanifold of $M$. If $M$ is Riemannian, complete, and simply connected, then $M$ is isometric to the product $M^{\prime} \times R$.

Proposition 7. If $B_{T} \equiv 0$ and $T$ is geodesic then $T$ has constant length $c$ and $\operatorname{Ric}(T)=-\operatorname{tr} A_{T}^{2}-T(\operatorname{div} T)$. When $M^{\prime}$ is defined it has total curvature zero and mean curvature $(1 / c) \operatorname{div} T$. If $M^{\prime}$ is defined and flat everywhere, then $A_{T} \equiv 0$ and Proposition 6 is applicable.

Proposition 8. If $B_{T} \equiv 0$ and the integral curves of $T$ are reparameterizations of geodesics with $D_{T} T=g T$, then at points where $g$ and $T$ do not vanish, $M^{\prime}$ is flat and the Ricci curvature of $T$ is zero.

In proving Proposition 8 one shows at points in $U$ where $g$ does not vanish then $A_{T} T=D_{T} T=(\operatorname{div} T) T$ by applying Proposition 6 to $D_{T} T$. Furthermore, at such points $0=B_{T} T=\left[T(\operatorname{div} T)+(\operatorname{div} T)^{2}\right] T$ so $\operatorname{tr} A_{T}^{2}=(\operatorname{div} T)^{2}=-T(\operatorname{div} T)$ and $\operatorname{Ric}(T)=0$.

Proposition 9. If $B_{T} \equiv 0$ and the integral curves of $T$ are not reparameterizations of geodesics, then Proposition 6 may be applied to $D_{T} T$. Moreover $T^{2}\langle T, T\rangle$ is constant, hence there can be at most one point on each integral curve of $T$ where the length of $T$ has a critical point. If the integral curves of $T$ are parametrically complete (defined for all parameter values), then $M$ cannot be compact.

Notice in Proposition 9 the length of $T$ is not constant along any of its integral curves, for $0=T\langle T, T\rangle=2\left\langle D_{T} T, T\right\rangle$ implies $D_{T} T=g T$ by Theorem 4 , which implies the integral curves of $T$ are geodesics by Proposition 5 . 


\section{BIBLIOGRAPHY}

1. S. Bochner, Vector fields and Ricci curvature, Bull. Amer. Math. Soc. 52 (1946), 776-797.

2. C. Chevalley, Theory of lie groups, Princeton Univ. Press, Princeton, 1949.

3. G. DeRham, Sur la reductibilité d'un espace de Riemann, Comment. Math. Helv. 26 (1952), 328-344.

4. S. Helgason, Differential geometry and symmetric spaces, Academic Press, New York, 1962.

5. R. Hermann, On geodesics that are also orbits, Bull. Amer. Math. Soc. 66 (1960), 91-93.

6. N. Hicks, Submanifolds of semi-Riemannian manifolds, Rend. Cir. Mat. Palermo, (to appear).

7. N. Hicks, Notes on differential geometry, D. Van Nostrand, Princeton, (to appear).

8. K. Nomizu, Lie groups and differential geometry, Math. Soc. Japan Publ. 2, 1956.

The University of MichigaN 



\section{PACIFIC JOURNAL OF MATHEMATICS}

\section{EDITORS}

H. Samelson

Stanford University

Stanford, California

R. M. Blumenthal

University of Washington

Seattle, Washington 98105
J. Dugundu

University of Southern California Los Angeles, California 90007

*Richard Arens

University of California

Los Angeles, California 90024

\section{ASSOCIATE EDITORS}
E. F. BECKENBACH
B. H. NeumanN
F. WOLF
K. YOSIDA

\section{SUPPORTING INSTITUTIONS}

UNIVERSITY OF BRITISH COLUMBIA CALIFORNIA INSTITUTE OF TECHNOLOGY UNIVERSITY OF CALIFORNIA MONTANA STATE UNIVERSITY

UNIVERSITY OF NEVADA

NEW MEXICO STATE UNIVERSITY

OREGON STATE UNIVERSITY

UNIVERSITY OF OREGON

OSAKA UNIVERSITY

UNIVERSITY OF SOUTHERN CALIFORNIA
STANFORD UNIVERSITY

UNIVERSITY OF TOKYO

UNIVERSITY OF UTAH

WASHINGTON STATE UNIVERSITY

UNIVERSITY OF WASHINGTON

AMERICAN MATHEMATICAL SOCIETY CALIFORNIA RESEARCH CORPORATION SPACE TECHNOLOGY LABORATORIES NAVAL ORDNANCE TEST STATION 


\section{Pacific Journal of Mathematics}

\section{Vol. 15, No. $1 \quad$ September, 1965}

Donald Charles Benson, Unimodular solutions of infinite systems of linear

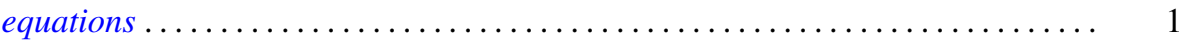

Richard Earl Block, Transitive groups of collineations on certain designs . . . . . . 13

Barry William Boehm, Existence of best rational Tchebycheff approximations .... . 19

Joseph Patrick Brannen, A note on Hausdorff's summation methods . . . . . . . . . . 29

Dennison Robert Brown, Topological semilattices on the two-cell ............ 35

Peter Southcott Bullen, Some inequalities for symmetric means . . . . . . . . . . 47

David Geoffrey Cantor, On arithmetic properties of coefficients of rational

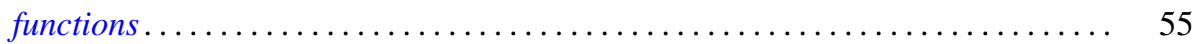

Luther Elic Claborn, Dedekind domains and rings of quotients . . . . . . . . . 59

Allan Clark, Homotopy commutativity and the Moore spectral sequence ........ 65

Allen Devinatz, The asymptotic nature of the solutions of certain linear systems of

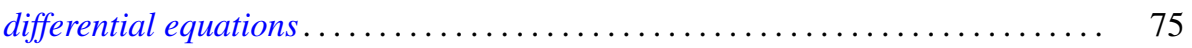

Robert E. Edwards, Approximation by convolutions ................... 85

Theodore William Gamelin, Decomposition theorems for Fredholm operators . . . . . 97

Edmond E. Granirer, On the invariant mean on topological semigroups and on

topological groups .................................. 107

Noel Justin Hicks, Closed vector fields . . . . . . . . . . . . . . . . . . . 141

Charles Ray Hobby and Ronald Pyke, Doubly stochastic operators obtained from

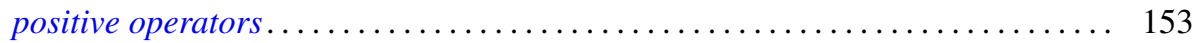

Robert Franklin Jolly, Concerning periodic subadditive functions . . . . . . . . . 159

Tosio Kato, Wave operators and unitary equivalence . . . . . . . . . . . . . . 171

Paul Katz and Ernst Gabor Straus, Infinite sums in algebraic structures . . . . . . . 181

Herbert Frederick Kreimer, Jr., On an extension of the Picard-Vessiot theory ...... 191

Radha Govinda Laha and Eugene Lukacs, On a linear form whose distribution is

identical with that of a monomial ......................... 207

Donald A. Ludwig, Singularities of superpositions of distributions . . . . . . . . . 215

Albert W. Marshall and Ingram Olkin, Norms and inequalities for condition

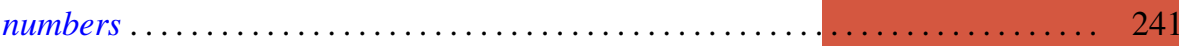

Horace Yomishi Mochizuki, Finitistic global dimension for rings . . . . . . . . . . 249

Robert Harvey Oehmke and Reuben Sandler, The collineation groups of division

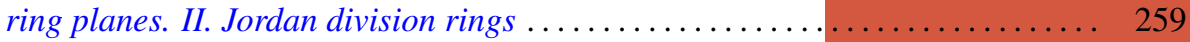

George H. Orland, On non-convex polyhedral surfaces in $E^{3} \ldots \ldots \ldots \ldots \ldots \ldots \ldots 267$

Theodore G. Ostrom, Collineation groups of semi-translation planes . . . . . . . . 273

Arthur Argyle Sagle, On anti-commutative algebras and general Lie triple

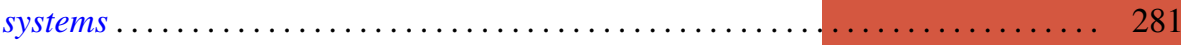

Laurent Siebenmann, A characterization of free projective planes . . . . . . . . . 293

Edward Silverman, Simple areas.................................. 299

James McLean Sloss, Chebyshev approximation to zero .................. 305

Robert S. Strichartz, Isometric isomorphisms of measure algebras . . . . . . . . . 315

Richard Joseph Turyn, Character sums and difference sets . . . . . . . . . . . . 319

L. E. Ward, Concerning Koch's theorem on the existence of arcs . . . . . . . . . . 347

Israel Zuckerman, A new measure of a partial differential field extension ......... 357 